\title{
LITIASIS URINARIA EN PEDIATRÍA
}

\author{
(URINARY LITHIASIS IN PEDIATRICS)
}

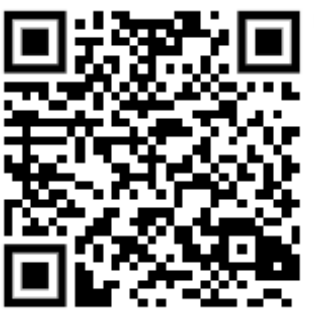

\author{
'Dra. Jenny Badilla García \\ Área de Salud Palmares, Alajuela, Costa Rica \\ badillajenny@outlook.com \\ https://orcid.org/0000-0002-2383-2083 \\ ${ }^{2}$ Dr. Jorge Andrés Herrera Corrales \\ Área de Salud Naranjo, Alajuela, Costa Rica \\ Jorherrera87@hotmail.com \\ https://orcid.org/0000-0002-1210-1728
}

RECIBIDO CORREGIDO

ACEPTADO

$7 / 12 / 2018$

$17 / 12 / 2018$

$21 / 12 / 2018$

\section{RESUMEN}

La litiasis renal es una entidad infrecuente en la infancia y su incidencia va en aumento en los países desarrollados. Afecta más a los individuos de raza blanca que a los afroamericanos en una proporción 4:1, con predominio del sexo masculino. Existen factores geográficos, raciales, genéticos implicados en su patogenia, que depende también de factores fisicoquímicos (eliminación renal de agua y solutos, $\mathrm{pH}$ urinario, equilibrio entre factores estimulantes/inhibidores de la cristalización), alteraciones anatómicas, infecciones y cambios socioeconómicos; los cuales a lo largo del tiempo han producido cambios en los hábitos dietéticos, lo que ha modificado la frecuencia, composición química y localización de los cálculos. A pesar de su rareza se debe pensar en la litiasis, con el fin de evitar un daño renal irreversible. La disponibilidad de terapia menos agresiva ha reducido a un $5 \%$ las indicaciones quirúrgicas, abriendo nuevas perspectivas en el tratamiento de la urolitiasis en la infancia.

${ }^{1}$ Médico general, graduada de la Universidad de Iberoamérica (UNIBE), médico del área de salud Palmares, Alajuela, Costa Rica.

Código médico: 13300 badillajenny@outlook.com

${ }^{2}$ Médico general, graduado de la Universidad de Costa Rica (UCR), médico del área de salud Naranjo, Alajuela, Costa Rica.

Código médico: 13193 Jorherrera87@hotmail.com

PALABRAS CLAVES: cálculos renales, hematuria, ultrasonografía, oxalato de calcio, litotricia. 
factors/inhibitors of crystallization), anatomical alterations, infections and socioeconomic changes. Over time, changes have occurred in dietary habits, which has changed the frequency, chemical composition and location of the stones. Despite its rarity, lithiasis must be considered in order to avoid irreversible kidney damage. The availability of less aggressive therapy has reduced surgical indications to $5 \%$, opening new perspectives in the treatment of urolithiasis in childhood.

KEYWORDS: kidney calculi, hematuria, ultrasonography, calcium oxalate, lithotripsy.

\section{INTRODUCCIÓN}

Desde la antigüedad existe evidencia de que el ser humano ha sufrido de urolitiasis, con casos reportados en momias de niños egipcios (1). La litiasis urinaria en los niños es multifactorial, se presenta en todo el mundo y tiende a recurrir $(2,3)$. Su incidencia en la población pediátrica es del $1-3 \%$ en los países desarrollados, siendo pequeña al compararla con la población adulta (510\%). Existen estudios recientes en Estados Unidos que sugieren un incremento de esta incidencia que oscila de 1/1.000 a 1/7.500 (4). La probabilidad de sufrir cálculos sintomáticos es 10 veces superior en los adolescentes en comparación con los niños de 0-3 años. La mayoría de los niños con litiasis padecen una alteración metabólica. La excepción la constituyen los pacientes con una vejiga neurógena, que son propensos a la formación de cálculos infecciosos, y aquéllos en los que se ha efectuado una reparación de las vías urinarias utilizando intestino, lo que predispone a la formación de cálculos vesicales (2). Estudio retrospectivo indicó una mayor incidencia entre los 5-10 años. La media de edad de presentación fue de 8,2 años, con un 54,4\% de varones. Se hallaron antecedentes familiares de litiasis en el $50 \%$ de los casos. Infecciones urinarias y alteraciones metabólicas (hipercalciuria, acidosis tubular, cistinuria) fueron los factores etiológicos más frecuentemente hallados. Se consideró litiasis idiopática en el $15 \%$ de los casos (5). Es una patología con alta morbilidad debido a la posibilidad de causar lesiones estructurales en el riñón o las vías urinarias en edades muy tempranas (6). En individuos sanos, la orina transita por el tracto urinario sin que se forman cristales o estos son tan pequeños que son eliminados de forma asintomática (cristaluria asintomática) (6).

\section{FORMACIÓN DE CÁLCULOS}

Alteraciones metabólicas predisponen a la formación de cálculos (2): la hipercalciuria es el factor metabólico de riesgo más reconocido mundialmente, seguida de la hipocitraturia, con menor frecuencia de la hiperuricosuria, hiperoxaluria, cistinuria heterocigótica, hipomagnesuria, el hiperparatiroidismo y la acidosis tubular renal $(2,7)$.

Aproximadamente el $90 \%$ de todos los cálculos contienen calcio como 
componente principal y el $60 \%$ están compuestos por oxalato cálcico (estudios epidemiológicos sugieren influencia genética en la hipercalciuria), otros están compuestos por ácido úrico, cistina, cristales de amonio o de fosfato o por una combinación de estas sustancias $(2,7)$.

El riesgo de formación de cálculos aumenta cuando lo hacen las concentraciones de estos cristales, y se reduce cuando aumentan las concentraciones de sustancias inhibidoras urinarias (2).

Los factores que favorecen la formación de cálculos urinarios son: el volumen urinario bajo, el pH urinario bajo, el calcio, el sodio, el oxalato y el urato.

Muchas sustancias inorgánicas (p. ej., citrato, magnesio) y orgánicas (p. ej., glucosaminoglucanos, osteopontina) inhiben la formación de cálculos. Los compuestos inhibitorios orgánicos se unen a la superficie del cristal, inhibiendo su crecimiento y su nucleación. La formación de cálculos depende de cuatro factores: matriz, precipitacióncristalización, epitaxia y ausencia en la orina de sustancias inhibidoras de la formación de cálculos. La matriz es una mezcla de proteínas, azúcares no aminados, glucosamina, agua y ceniza orgánica.

Constituye el $2-9 \%$ del peso en seco de los cálculos urinarios y se dispone en su interior formando estructuras laminares concéntricas.

La precipitación-cristalización hace referencia a la sobresaturación de la orina para los iones específicos que componen el cristal.

El aumento de la saturación urinaria para los iones incrementa el ritmo de nucleación, el crecimiento de los cristales, su agregación, aumenta la probabilidad de formación y crecimiento de cálculos.

La epitaxia consiste en la agregación de cristales de composiciones diferentes, pero con una estructura reticular similar; se forman así cálculos de naturaleza heterogénea.

\section{MANIFESTACIONES CLÍNICAS}

El cuadro clínico de la litiasis urinaria depende de la edad del niño, el tamaño y la localización del cálculo $(3,7)$. Los niños presentan habitualmente hematuria macroscópica o microscópica y dolor abdominal inespecífico $(2,7)$. También pueden encontrarse signos y síntomas generales como nauseas, vómitos, manifestaciones de disfunciones miccionales caracterizadas por pérdidas urinarias diurnas o nocturnas y urgencia miccional (7).

Si el cálculo provoca obstrucción, se produce dolor importante en la fosa lumbar (cólico renal) o en el abdomen. El cálculo típicamente causa obstrucción en zonas de estrechamiento de las vías urinarias: la unión pieloureteral, en la zona en la que el uréter cruza los vasos ilíacos, y la unión ureterovesical.

El uréter se estrecha progresivamente en dirección distal, y su segmento más estrecho es la unión ureterovesical. Habitualmente, el dolor se irradia en dirección anterior hacia el escroto o los labios.

A menudo el dolor es intermitente, en correspondencia con períodos de obstrucción del flujo urinario, que aumentan la presión en el sistema colector.

Si el cálculo está en el uréter distal, el niño puede presentar síntomas irritativos 
como disuria, tenesmo y polaquiuria. Cuando el cálculo pasa a la vejiga, el niño suele permanecer asintomático. Si el cálculo está en la uretra puede producirse disuria y dificultad miccional, sobre todo en los varones.

Algunos niños eliminan pequeñas cantidades de material tipo arenilla (2).

\section{DIAGNÓSTICO}

La litiasis es tan solo la manifestación de otras enfermedades subyacentes, pero no la enfermedad en si, por lo que se necesita una evaluación diagnóstica completa en cada caso, de forma que se pueda instaurar el tratamiento adecuado lo más temprano posible. El historial médico debe incluir toda la información posible sobre antecedentes familiares (50\% de los pacientes tienen familiar con urolitiasis y nos orienta sobre posible trastorno genético), prematuridad, enfermedades concomitantes (con su tratamiento médico), ingesta diaria de líquidos, dieta, uso de suplementos vitamínicos $(1,8)$.

El diagnóstico puede ser casual al realizar una ultrasonografía abdominal en un niño asintomático, o puede realizarse en un paciente con síntomas clínicos en el que ya se sospecha la enfermedad litiásica (3).

Para la confirmación diagnóstica de la litiasis urinaria son necesarios exámenes de laboratorio y estudios por imagen. Se recomienda la realización de los siguientes exámenes en la fase aguda: el estudio del perfil metabólico en sangre y orina para determinar la etiopatogenia del cálculo; urocultivo (para la identificación de los cálculos asociados con infecciones urinarias), antibiograma en caso necesario, radiografías simples de abdomen sin preparación (para evaluación de la ubicación y el tamaño del cálculo), en general mediante ecografía del aparato urinario y se utiliza tomografía computarizada de los riñones/vías urinarias en casos seleccionados (7). Una vez que se establece la presencia de litiasis, lo importante es precisar la alteración metabólica que condiciona la formación de cálculos (2). Aproximadamente el $90 \%$ de los cálculos urinarios están más o menos calcificados, por lo que son radiopacos en la radiografía simple de abdomen. No obstante, muchos sólo tienen unos pocos milímetros de diámetro y pueden pasar inadvertidos, especialmente si están en el uréter. Los cálculos de estruvita (fosfato amónico magnésico) son radiopacos. Los cálculos de cistina, xantina y ácido úrico pueden ser radiotransparentes, pero a menudo están ligeramente opacificados.

Algunos niños presentan nefrocalcinosis o calcificación del tejido renal. La nefrocalcinosis se observa más frecuentemente en recién nacidos prematuros tratados con furosemida, que causa hipercalciuria, y en niños con espongiosis medular renal. Hay numerosas opciones de imagen en los niños en los que se sospecha un cólico renal. El estudio de mayor precisión diagnóstica es la TC helicoidal sin contraste del abdomen y la pelvis; delimita el número, también la localización de los cálculos con una sensibilidad y una especificidad del $96 \%$. Además demuestra si el riñón afectado es hidronefrótico; Sin embargo, la exposición radiactiva es alta. Otra alternativa es obtener una radiografía 
simple de abdomen, pelvis y una ecografía renal. Estos estudios pueden demostrar la existencia de hidronefrosis y posiblemente del cálculo; sin embargo, el cálculo no se visualiza en la ecografía a menos que esté situado junto a la vejiga. Además, los cálculos renales $<3$ $\mathrm{mm}$ típicamente no son visibles. Así pues, los médicos necesitan analizar cuidadosamente los riesgos de la TC, frente a la menor sensibilidad de la radiografía abdominal simple más ecografía. En un niño con un cálculo ya diagnosticado, las radiografías simples seriadas o la ecografía renal pueden emplearse para controlar el estado del cálculo, si ha aumentado o disminuido de tamaño o si se ha desplazado. Cualquier material con aspecto de cálculo debe ser enviado para su análisis a un laboratorio especializado en la identificación de los componentes de los cálculos urinarios (2).Es muy importante advertir a los padres que cuando se va a efectuar el análisis de sustancias litogénicas e inhibidoras del lito, no se debe aumentar drásticamente la ingesta de líquidos. Esto debido a que un aumento excesivo en la ingesta de líquidos, y por lo tanto en el volumen de orina, puede enmascarar el verdadero factor de riesgo relacionado con el paciente. Por ejemplo, muchas niñas pequeñas tienen solo un factor de riesgo identificable: baja ingesta de líquidos. Por lo tanto, un aumento en la ingesta de líquidos justo en el momento de la (s) recolección (es), probablemente va a imposibilitar dilucidar el motivo de la urolitiasis (9).

\section{ESTUDIO METABÓLICO}

En todos los niños con urolitiasis debe efectuarse una valoración metabólica de los factores predisponentes más frecuentes, teniendo en cuenta que a menudo coexisten factores estructurales, infecciosos y metabólicos. Esta valoración no debe iniciarse en un niño en pleno proceso de eliminación de un cálculo, puesto que el cambio de dieta, el estado de hidratación, así como el efecto de la obstrucción sobre el riñón, pueden alterar los resultados del estudio (2).

Los cálculos vesicales asociados con desnutrición infantil, se forman preferentemente con las dietas basadas en cereales, aún son endémicas en Turquía, China, India, y el continente africano. En el noreste de Tailandia, la elevada endemicidad de la urolitiasis se asoció con la alta prevalencia de acidosis tubular e hipocitraturia. Por otro lado en el norte de la India la ausencia de una bacteria intestinal que degrada el oxalato, el oxalobacter formigenes, ha sido implicado en la alta incidencia de hiperoxaluria absortiva, con formación de oxalato de calcio vista en esta región del mundo. En EEUU, la prevalencia de los cálculos urinarios se eleva en dirección norte-sur y oeste- este; el $75 \%$ de estos niños tienen un factor de riesgo identificable y la hipercalciuria 0 la hiperuricosuria se identifica en el $50 \%$ de los casos (7).

\section{PATOGENIA DE LOS DIFERENTES TIPOS DE CÁLCULOS RENALES}

En los cálculos de oxalato cálcico y de fosfato cálcico la anomalía metabólica más frecuente en estos niños es la hipercalciuria normocalcémica. La hipercalciuria puede ser absortiva, renal o resortiva. El trastorno primario en la hipercalciuria absortiva es la hiperabsorción intestinal de calcio. En 
algunos niños, un aumento de la 1,25dihidroxivitamina $D$ se asocia a un aumento de la absorción de calcio; mientras que en otros el proceso es independiente de la vitamina D. La hipercalciuria renal consiste en la alteración de la reabsorción tubular renal de calcio. Las pérdidas renales de calcio provocan una hipocalcemia moderada, que induce un aumento de la producción de hormona paratiroidea, con incremento de la absorción intestinal de calcio y de la movilización de los depósitos cálcicos. La hipercalciuria resortiva es poco frecuente y se observa en pacientes con un hiperparatiroidismo primario.

exceso de secreción de hormona paratiroidea estimula la absorción intestinal de calcio y la movilización de los depósitos cálcicos. La hiperoxaluria es otra causa potencialmente importante de cálculos de calcio. El oxalato aumenta de 7 a 10 veces más que el calcio el producto de solubilidad en la cristalización del oxalato cálcico. Por tanto, la hiperoxaluria aumenta significativamente la probabilidad de precipitación del oxalato cálcico. El oxalato aparece en altas concentraciones en el té, el café, las espinacas y el ruibarbo. La hiperoxaluria primaria es un trastorno autosómico recesivo infrecuente que puede subdividirse en aciduria glucólica y aciduria I-glicérica. La mayoría de los pacientes con hiperoxaluria primaria tienen aciduria glucólica; en los individuos afectados hay un aumento de los ácidos oxálico y glucólico en la orina. Ambos defectos provocan un aumento de la producción endógena de oxalato, con hiperoxaluria, urolitiasis, nefrocalcinosis y lesión renal. En los pacientes no tratados, la muerte sobreviene hacia los 20 años por insuficiencia renal. La oxalosis, que se define como el depósito extrarrenal de oxalato cálcico, se produce cuando existe insuficiencia renal con un aumento del oxalato plasmático. Los depósitos de oxalato cálcico aparecen en primer lugar en los vasos sanguíneos, la médula ósea, y con el tiempo se observan en todo el cuerpo. La hiperoxaluria secundaria es más frecuente, puede producirse en pacientes con un aumento de la ingesta de oxalatos, de sus precursores, como la vitamina $\mathrm{C}$, en aquellos que padecen déficit de piridoxina y en niños con malabsorción intestinal.

La hiperoxaluria entérica abarca trastornos como la enfermedad inflamatoria intestinal, la insuficiencia pancreática, las enfermedades biliares, en los que existe una malabsorción gastrointestinal de ácidos grasos, que se unen al calcio intraluminal y forman sales que se excretan en las heces. Normalmente, el calcio forma un complejo con el oxalato y reduce la absorción de éste, pero, si no hay calcio disponible, se produce un aumento de la absorción de oxalato libre. La hipocitraturia consiste en una excreción baja de citrato, que es un inhibidor de la formación de cálculos de calcio. El citrato actúa como un inhibidor de la urolitiasis cálcica al formar complejos con el calcio, aumentar la solubilidad del calcio en la orina e inhibir la agregación de los cristales de fosfato cálcico y oxalato cálcico. Algunos trastornos como la diarrea crónica, la malabsorción intestinal y la acidosis tubular renal pueden provocar hipocitraturia. También puede 
ser idiopática. La acidosis tubular renal (ATR) es un síndrome en el que existe un trastorno del equilibrio acido básico renal. Puede clasificarse en 3 tipos, uno de los cuales predispone a los cálculos renales, típicamente de fosfato cálcico. En la ATR de tipo 1, la nefrona distal no segrega iones hidrógeno en el túbulo distal. El pH urinario nunca es inferior a 5,8 y se produce acidosis hiperclorémica hipopotasémica. Los pacientes presentan nefrolitiasis, nefrocalcinosis, debilidad muscular y osteomalacia. La ATR de tipo 1 puede ser un trastorno autosómico dominante, pero con mayor frecuencia es adquirido y se asocia a enfermedades sistémicas como el síndrome de Sjögren, la enfermedad de Wilson, la cirrosis biliar primaria, la tiroiditis linfocítica, 0 se debe a anfotericina $B$, litio 0 tolueno (un disolvente orgánico asociado a la inhalación de pegamentos) (2).

El $5-8 \%$ de los pacientes con fibrosis quística presenta urolitiasis. Típicamente, los cálculos son de calcio y a menudo aparecen en adolescentes. En los niños pequeños que padecen esta enfermedad también se produce una nefrocalcinosis microscópica. Estos pacientes no presentan hipercalciuria, y se cree que la propensión a la urolitiasis se debe a la incapacidad para excretar un exceso de cloruro sódico 0 a la malabsorción intestinal. Existen otros trastornos que pueden desempeñar un cierto papel en la formación de cálculos de calcio. La hiperuricosuria puede estar relacionada con el crecimiento epitáxico de cristales de oxalato cálcico alrededor de un núcleo de cristales de ácido úrico o con la acción del ácido úrico como antagonista de los mucopolisacáridos urinarios, que inhibe la cristalización del oxalato cálcico. La cistinuria heterocigótica se observa en algunos pacientes con cálculos de calcio. El mecanismo de acción es desconocido, pero puede ser similar al del ácido úrico. La sarcoidosis provoca un aumento de la sensibilidad a la vitamina D3, por lo que aumenta la absorción de calcio en el sistema digestivo. En el síndrome de Lesch-Nyhan existe una síntesis excesiva de ácido úrico. Estos pacientes son más propensos a la formación de cálculos de ácido úrico, aunque alguno de estos cálculos puede estar calcificado. En algunos niños, los cálculos de calcio son idiopáticos. Este diagnóstico sólo debe establecerse tras una valoración metabólica completa (2).

\section{- Cálculos de cistina}

Causa el $1 \%$ de los cálculos renales en niños. Se trata de un raro trastorno autosómico recesivo de las células epiteliales del túbulo renal que impide la absorción de los cuatro aminoácidos dibásicos (cistina, ornitina, arginina y lisina), provoca una excesiva excreción urinaria de estos productos. La única complicación conocida de esta enfermedad familiar es la formación de cálculos, debido a la baja solubilidad de la cistina. Los pacientes tienen habitualmente una orina ácida, lo que aumenta la tasa de precipitación. El contenido de azufre presente en la cistina hace que estos cálculos sean débilmente radiopacos (2).

\section{- Cálculos de estruvita}

La prevalencia de cálculos de estruvita asociados con infección, elevada en el pasado, hoy en día es menor en la mayoría de países industrializados, probablemente por el mejor diagnóstico y tratamiento de las uropatías obstructivas, 
también de las infecciones urinarias pediátricas (7). Las infecciones urinarias causadas por gérmenes productores de ureasa (más frecuentemente Proteus spp, en ocasiones, Klebsiella spp., Escherichia coli, Pseudomonas spp. entre otros) provocan una alcalinización de la orina y un exceso de producción de amoníaco; lo que puede llevar a la precipitación de fosfato amónico magnésico (estruvita) y fosfato cálcico. En el riñón, los cálculos presentan a menudo un aspecto coraliforme y ocupan los cálices. Actúan como cuerpos extraños, provocan obstrucción, perpetúan la infección y causan lesión renal progresiva. Los pacientes con cálculos de estruvita presentan también anomalías metabólicas que predisponen a la formación de cálculos. Estos cálculos se observan a menudo en niños con vejiga neurógena, particularmente en aquellos a los que se les ha practicado una intervención reparadora de las vías urinarias. También se pueden formar cálculos de estruvita en la vejiga reparada de los niños a los que se les ha realizado una cistoplastia de ampliación o una derivación del continente urinario.

\section{- Cálculos de ácido úrico}

En EEUU representan menos del 5\%, pero en otras zonas del mundo menos desarrolladas son más frecuentes. La hiperuricosuria, con hiperuricemia o sin ella, es el factor subyacente más común en la mayoría de los casos. El diagnóstico debe sospecharse cuando la orina es persistentemente ácida y contiene cristales de urato. La hiperuricosuria puede ser la consecuencia de diferentes trastornos congénitos del metabolismo de las purinas, que dan lugar a un exceso de producción de ácido úrico, el producto final del metabolismo de las purinas en los seres humanos. Los niños con el síndrome de Lesch- Nyhan y los pacientes con deficiencia de glucosa-6fosfatasa también forman cálculos de urato. En los niños con síndrome del intestino corto, especialmente en aquellos a los que se les ha practicado una ileostomía, la deshidratación crónica y la acidosis se complican a veces con litiasis de ácido úrico.

Una de las causas más frecuentes de litiasis de ácido úrico es el rápido recambio de purinas en algunos tumores y enfermedades mieloproliferativas. El riesgo de litiasis de ácido úrico es especialmente importante cuando el tratamiento de estas enfermedades provoca una rápida destrucción de nucleoproteínas. Los cálculos o el barro de ácido úrico pueden ocupar toda la parte alta del sistema colector y provocar insuficiencia renal o incluso anuria. Los uratos también pueden aparecer en el interior de cálculos que contienen calcio. En tales casos, puede que exista más de un factor predisponente para la formación de cálculos (2). La base del tratamiento es la alcalinización de la orina, en principio usando citrato de potasio, también se aconseja reducir el aporte dietético de purinas, y en casos seleccionados, agregar alopurinol por vía oral $10 \mathrm{mg} / \mathrm{kg} /$ días una vez por día o dividido en 2 o 3 veces (7). El alopurinol es eficaz en pacientes con cálculos de ácido úrico; es un inhibidor de la xantina oxidasa que reduce eficazmente la producción de ácido úrico y de 2,8dihidroxiadenina (2). 
Si a pesar del control bioquímico y de alcalinizar un $\mathrm{pH}$ urinario optimo el paciente sigue produciendo cálculos urinarios, se debe sospechar la presencia de cálculos de xantina, metabolitos de ácido úrico debidos a alopurinol (7).

\section{- Cálculos de indinavir}

El sulfato de indinavir es un inhibidor de la proteasa aprobado para el tratamiento de la infección por VIH. Hasta un $4 \%$ de los pacientes tratados con indinavir desarrollan nefrolitiasis sintomática. La mayoría de los cálculos son radiotransparentes y están compuestos por monohidrato de indinavir, aunque en algunos se observa la presencia de oxalato o fosfato cálcico. Después de cada dosis, el $12 \%$ del fármaco se excreta por la orina sin ser modificado. La orina de estos pacientes contiene a menudo cristales con una forma característica rectangular, en abanico o en explosión estelar. El indinavir es soluble a un $\mathrm{pH}$ inferior a 5,5.

Por tanto, debe considerarse la posibilidad de un tratamiento basado en su disolución mediante la acidificación de la orina con cloruro amónico o ácido ascórbico (2).

\section{- Nefrocalcinosis}

La nefrocalcinosis consiste en el depósito de calcio en el tejido renal. A menudo la nefrocalcinosis se acompaña de urolitiasis. Sus causas más frecuentes son la furosemida, la acidosis tubular renal distal, el hiperparatiroidismo, la espongiosis medular renal, el raquitismo hipofosfatémico, la sarcoidosis, la necrosis cortical, la hiperoxaluria, la inmovilización prolongada, el síndrome de Cushing, la hiperuricosuria, las causas monogenéticas de hipertensión y la candidiasis renal (2).

\section{- Cálculos con infección}

Estos deben extraerse quirúrgicamente una vez controlado el episodio infeccioso. La prevención de las recidivas se logra corrigiendo las anormalidades anatómicas y usando quimioprofilaxis con nitrofurantoina 0 trimetoprima/sulfametoxazol en dosis habituales (7). En un estudio retrospectivo con 112 pacientes pediátricos con litiasis renal existía historia de infección urinaria en la tercera parte de los pacientes, y urocultivo positivo en el $25 \%$ de ellos en el momento del ingreso. El germen más frecuente fue Proteus (55\%), siendo también el organismo implicado con mayor frecuencia en los casos de pielonefritis aguda asociada a la litiasis $(57 \%)(5,7)$.

\section{TRATAMIENTO}

Cada alteración conlleva medidas terapéuticas diferentes (3).

En la fase aguda: el alivio puede lograrse con fármacos antiespasmódicos y analgésicos antiinflamatorios no esteroideos $u$ opioides $(2,7)$. En esta fase, los antinflamatorios no esteroideos han demostrado ser superiores incluso a los opioides. Hay que tener precaución al usar los antiespasmódicos en el periodo expulsivo, ya que estos inhiben el peristaltismo ureteral, y aumentan el íleo intestinal a través de un reflejo vagal (10).

Si el cuadro agudo se acompaña de vómitos, se indican agentes antieméticos e hidratación intravenosa. 
La migración del cálculo debe confirmarse con imágenes periódicas, preferiblemente ecografía de las vías urinarias (7).

Los cálculos ureterales pequeños suelen eliminarse espontáneamente, y pueden presentarse desde indoloros, hasta con cólico nefrítico intenso. Se ha demostrado que el tratamiento con antagonistas alfa-adrenérgicos, tamsulosina, terazosina o la doxazosina, facilita la eliminación de los cálculos; al reducir tanto la presión ureteral por debajo del cálculo, como la frecuencia de las contracciones peristálticas del uréter obstruido.

En muchos casos, la introducción endoscópica de una endoprótesis ureteral, hasta sobrepasar el cálculo, alivia el dolor y dilata suficientemente el uréter como para permitir la eliminación del cálculo. En los niños con un cálculo de ácido úrico o en lactantes con un cálculo debido a la administración de furosemida, el tratamiento basado en la disolución del cálculo puede ser eficaz.

Si el cálculo no se elimina o es poco probable que lo haga, o si hay una infección urinaria asociada, es necesaria su extirpación. En los cálculos vesicales, ureterales y en cálculos pequeños de la pelvis renal, la litotricia con láser de holmio a través de un ureteroscopio flexible o rígido es efectiva. La litotricia extracorpórea con ondas de choque (LEOC) se ha aplicado con éxito a los niños con cálculos renales y ureterales, con una tasa de resultados favorables superior al 75\% (2,7). Estudio retrospectivo demostró que las complicaciones fueron más frecuentes con el tratamiento quirúrgico que cuando se pudo emplear la litotricia, principalmente infecciones y complicaciones inherentes a la cirugía (estenosis postquirúrgicas) $(5,7)$. El tratamiento de la litiasis renal en niños mediante LEOC es un tratamiento seguro y eficaz (11).

Otra alternativa es la nefrostolitotomía percutánea, en la cual se accede al sistema colector percutáneamente, y los cálculos se rompen mediante litotricia ultrasónica. Cuando estas modalidades no consiguen el efecto deseado, una alternativa es la extirpación laparoscópica; este procedimiento puede llevarse a cabo con el robot da Vinci (2). La hidratación es la medida más importante, cuyo objetivo es disminuir la concentración de las sustancias litogenas en la orina, debe distribuirse a lo largo del día y la noche, esto para mantener un flujo urinario constante. El aporte de líquido en el niño debe de ser el suficiente para promover una diuresis de alrededor de $1 \mathrm{ml} / \mathrm{kg} / \mathrm{h}$. Se recomienda de 2-2,5 $L$ diarios en adolescentes, aumentando dicha ingesta durante los meses de verano $(2,7)$. La reposición de líquidos después de la actividad física debe ser sistemática, para impedir la concentración y saturación de la orina. Se sugiere ingerir preferiblemente líquidos con $\mathrm{pH}$ alcalino. La dieta debe ser adecuada a las necesidades del niño o del adolescente especialmente en la ingesta de calcio, proteínas, hidratos de carbono, grasas y sales. Se recomienda que el plan dietético sea individualizado y realizado por un nutricionista (7).

La ingesta excesiva de sodio aumenta la excreción urinaria de calcio y puede dar lugar a hipocitraturia.

Además, un mayor consumo de sal induce acidosis metabólica y para 
compensar la sobrecarga ácida, los riñones conservan aniones, incluido citrato urinario, lo que contribuye a la hipocitraturia. Por estas razones está indicado reducir la ingesta diaria de sodio y aumentar la ingesta de potasio.

Aunque es contraintuitivo, las dietas bajas en calcio son menos eficaces para el tratamiento de los cálculos de calcio que las dietas que contienen cantidades normales de calcio, cantidades reducidas de sodio y proteínas animales.

Las dietas bajas en sodio y proteínas reducen la excreción urinaria de calcio y oxalato. Deben evitar una ingesta excesiva de calcio. Sin embargo, los niños necesitan calcio para el desarrollo óseo y las recomendaciones sobre la ingesta diaria de calcio varían en función de la edad. Por tanto, en los niños se debe evitar la restricción de calcio. Los diuréticos tiazídicos también reducen la excreción renal de calcio. $\mathrm{La}$ administración de citrato potásico, un inhibidor de los cálculos de calcio, a dosis de 1-2 $\mathrm{mEq} / \mathrm{kg} / 24 \mathrm{~h}$, resulta beneficiosa. La limonada es una excelente fuente de citratos, puesto que unos $120 \mathrm{ml}$ de zumo de limón contienen $84 \mathrm{mEq}$ de ácido cítrico. La toma diaria de unos $120 \mathrm{ml}$ de zumo de limón disuelto en 2 litros de agua, aumenta significativamente la concentración urinaria de citratos.

$\mathrm{Si}$ se mantiene un $\mathrm{pH}$ urinario alto (mayor a 6,5) puede prevenirse la recurrencia de los cálculos de cistina. La cistina es mucho más soluble cuando el $\mathrm{pH}$ urinario es superior a 7,5. La dpenicilamina, es un quelante que se une a la cisteína o a la homocisteína y aumenta la solubilidad del producto. Aunque mal tolerada por muchos pacientes, se ha descrito su eficacia en la disolución de los cálculos de cistina también en la prevención de las recurrencias cuando fracasan la hidratación y la alcalinización urinaria.

El tratamiento de la acidosis tubular renal tipo 1, exige la corrección de la acidosis metabólica, la reposición de las pérdidas de potasio y sodio. Es necesario el tratamiento con citrato sódico y/o citrato potásico. Cuando se corrige la acidosis metabólica, la excreción urinaria de citrato vuelve a ser normal (2). Como se ha mencionado existen muchas opciones de tratamiento eficaces para la prevención los cálculos (12).

La litiasis tiene indicación quirúrgica cuando el dolor es intratable, hay obstrucción, infección asociada o una imposibilidad de eliminación espontanea del cálculo (7).

\section{CONCLUSIONES}

La urolitiasis es una enfermedad recurrente para la cual aún no se conoce cura (7). En esta revisión se ha tratado de llevar a los lectores, las principales causas de litiasis del tracto urinario en el niño, las medidas preventivas para evitar la formación de los cálculos, y la conducta que se debe adoptar de acuerdo con el tipo de trastorno metabólico del paciente. Por ser la causa más frecuente, se insiste en el diagnóstico y tratamiento de la litiasis producida por hipercalciuria idiopática (3).

La litiasis urinaria es un problema de salud relevante en la población infantil dado el rol que puede tener en la etiología de la infección urinaria y en el deterioro progresivo de la función renal. El riesgo de daño renal e insuficiencia renal crónica por litiasis urinaria es bajo, 
sin embargo algunos tipos de cálculos tienen un riesgo mayor como son los de origen hereditario (cistinuria, hiperoxaluria primaria, enfermedad de
Dent), las urolitiasis relacionadas con infección, las anomalías anatómicas o funcionales del tracto urinario o de la médula espinal

\section{REFERENCIAS}

1. Lagomarsino E, Ávila D, Baquedano P, Cavagnaro F, Céspedes P. Litiasis urinaria en pediatría. Revista chilena de pediatría. 2003;74(4):381-8. https://doi.org/10.4067/S0370-41062003000400005

2. Kliegman R, Stanton B, St Geme J, Shor N. Nelson Tratado de Pediatría. 20. Barcelona: ELsevier; 2016.

3. Durán Álvarez S. Urolitiasis en el niño. Revista Cubana de Pediatría. 2013;85(3):371-85.

4. Montalvo C, Gómez A. Tratamiento de la urolitiasis pediátrica en nuestro medio. Cir Pediatr. 2013;26(3):119-23.

5. Camacho Díaz J, Casas Gómez J, Amat Barnés A, Giménez Llort A, García García L. Litiasis renal en el niño. Anales españoles de pediatria. 1996;44(3):225-8.

6. Rodrigo M, Vicente C. Litiasis renal e hipercalciuria Idiopática. Protoc diagn ter pediatr. 2014;:150-70.

7. Paris E, Sánchez I, Beltramino D, Copto A. Meneghello. 6. Argentina: Panamericana; 2014.

8. Habbig S, Beck B, Hoppe B. Nephrocalcinosis and urolithiasis in children. Kidney Int. 2011 Sept 28;80(12):1279-91. https://doi.org/10.1038/ki.2011.336

9. Sáez-Torres C, Rodrigo D, Grases F, García-Raja AM, Gómez C, Lumbreras J, et al. Urinary excre $\neg$ tion of calcium, magnesium, phosphate, citra-te, oxalate and uric acid by healthy schoolchil $\neg$ dren following a 12-h collection protocol. Pediatric nephrology. 2014;29:1201-8. https://doi.org/10.1007/s00467-014-2755-1

10. Susaeta R, Benavente $D$, et al. Diagnóstico y manejo de litiasis renales en adultos y niños. Revista Médica Clínica Las Condes. 2018;29(2):197-212. https://doi.org/10.1016/j.rmclc.2018.03.002

11. J.M. Alapont Alacreu, J.A. Queipo Zaragoza, J.P. Burgués Gasión, E. Broseta Rico, A. Serrano Durbá, F. Boronat Tormo, J.F. Jiménez Cruz. Tratamiento con litotricia extracorpórea por ondas de choque en niños: nuestra experiencia. Suscribirse a:Actas Urológicas Españolas. 2002;26(1):15-9. https://doi.org/10.1016/S0210-4806(02)72722-8

12. Morgan MS, Pearle MS. Medical management of renal stones. British Medical Journal. 2016;352(8049):52. https://doi.org/10.1136/bmj.i52 